\title{
Inflection and Reflection: Design Issues that Shape the Field of Prevention Science
}

\author{
Catherine P. Bradshaw ${ }^{1}$
}

Published online: 16 September 2015

(C) Society for Prevention Research 2015

The field of prevention science has seen considerable change over the past decade, as both the rigor of the science has improved and the methods for assessing impact have expanded. A major force driving those innovations and advancements has been the Society for Prevention Research's standards of evidence, which was published in the journal Prevention Science by Flay and colleagues in 2005. In the current issue, we are pleased to feature an update to, and expansion on, that seminal work, which was led by Denise Gottfredson and colleagues (2015) at the request and endorsement of the Society for Prevention Research's Board of Directors.

I had the great fortune of coming into my role as editor of the journal just as this "next generation" effort was being launched. I was witness to the great strides that Denise and colleagues took to carefully review the literature and reflect on the highest standards of the field. Some may view these standards as aspirational, whereas others may consider them guideposts for the field. What is clear is the level of thought and reflection that went into crafting these updated and expanded standards. I anticipate that this paper will prove to be another inflection point for the field and shape research over the next decade and beyond.

We gain further insight into the potential impacts of this work through three invited commentaries. Of particular interest is the insightful reflection by Anthony Biglan, Brian Flay, and Alex Wagenaar; their comments are especially relevant, given both Brian and Anthony were leading authors on the 2005 standards. The two other invited commentaries, one by

Catherine P. Bradshaw

cpb8g@eservices.virginia.edu

1 University of Virginia, Charlottesville, VA, USA
Leonard Bickman and the other by Lauren Supplee and Aleta Meyer, provide additional perspectives on the scope and potential policy relevance of the new standards. It is our hope that the release of these new standards promotes further discussion and reflection on the methods and science that drive our collective work and the broader field of prevention research.

Also in this issue, we are pleased to feature a special section on research methods, with a particular focus on small samples and health disparities research. The former editor of Prevention Science, Robert McMahon, worked closely with guest editors Carlotta Ching Ting Fok, David Henry, and James Allen to organize and edit this special section. Robert and I thought this inspiring collection of methodological and design relevant papers and commentaries was a nice complement to the new standards. Therefore, we decided to include both sets of timely work in this expanded issue of the journal.

As we were finalizing the production of this important issue of Prevention Science, we were incredibly sad to learn of the passing of David Henry, who had worked tirelessly as guest editor on the special section while struggling with his illness. As a result, we felt it was appropriate to dedicate this issue to David in honor not only of his leadership on this collection of papers but for his broader contributions to the field. As reflected in this special section, David's work focused on the intersection of conceptual and methodological innovations, always with a developmental-ecological frame and great appreciation for the practical impact of research. He made numerous important substantive and methodological contributions that advanced the quality and impact of prevention science, developmental and ecological understanding of risk, and intervention evaluation. His contributions were recognized by the Society for Prevention Research through both the Nan Tobler Award in 2012 and the Community, Culture and Prevention Award in 2013. I recently had the great fortune of co-authoring a paper with him focused on the design and 
evaluation of community-focused youth violence prevention efforts. I learned a lot from him through that experience, as well as through the numerous reviews he did for the journal. While this moment serves as an important inflection point for prevention science, it also allows us to reflect on some of the great scholars who have helped shape the field and laid the foundation for the next generation.

Conflict of Interest The author declares that she has no competing interests.

\section{References}

Flay, B. R., Biglan, A., Boruch, R. F., Gonzalez Castro, F., Gottfredson, D., Kellam, S., Moscicki, E. K., Schinke, S., Valentine, J. C., \& Ji, P. (2005). Standards of evidence: Criteria for efficacy, effectiveness and dissemination. Prevention Science, 6, 151-175.

Gottfredson, D. C., Cook, T. D., Gardner, F. E. M., Gorman-Smith, D., Howe, G. W., Sandler, I. N., \& Zafft, K. M. (2015). Standards of evidence for efficacy, effectiveness, and scale-up research in prevention science: Next generation. Prevention Science. doi:10.1007/ s11121-015-0555-x. 